\title{
Control in vitro de garrapatas (Boophilus microplus; Acari: Ixodidae) mediante extractos vegetales
}

\author{
Víctor Álvarez ${ }^{1}$, Jorge Loaiza ${ }^{2}$, Roberto Bonilla ${ }^{1} \&$ Mariano Barrios $^{2}$ \\ 1 Ministerio de Agricultura y Ganadería, Servicio Nacional de Salud Animal, 11965-1000, San José, Costa Rica; \\ viacal@racsa.co.cr; rbonilla@protecnet.go.cr \\ 2 Universidad Nacional, Escuela de Química, Heredia, Costa Rica; jloaiza@una.ac.cr; mbarrios@una.ac.cr
}

\author{
Recibido 25-VIII-2006. Corregido 16-V-2007. Aceptado 27-VII-2007.
}

\begin{abstract}
Control in vitro tick (Boophilus microplus; Acari: Ixodidae) through plant extracts. Hydro-alcohol extracts of ten tropical plants were tested by the immersion method on mature ticks (Boophilus microplus Canestrini 1887). The biological evaluation was made with raw hydro alcoholic extracts (R-OH) to determine the most promissory compounds, which later were fractioned in their polar and non-polar parts. The polar parts showed a significantly inferior effect. The species that produced the highest mortality were Zizigium aromaticum, Morus alba, Piper nigrum and the mixture of Allium sativum- Z. aromaticum (all non-polar); on oviposition: $M$. alba, P. nigrum and the mixture of A. sativum - Z. aromaticum (all non-polar); on eclosion: $P$. nigrum, Z. aromaticum, Echinacea angustiofilia, Poligonum punctatum, M. alba and the mixture of A. sativumZ. aromaticum (all non-polar): on control percentage: P. nigrum, E. angustifolia, P. punctatum and M. alba (all non-polar, except for M. alba in which both fractions showed important activity); and finally, on the percentage of oviposition inhibition: P. nigrum, E. angustifolia, P. punctatum, Gliricidia sepium and M. alba. Rev. Biol. Trop. 56 (1): 291-302. Epub 2008 March 31.
\end{abstract}

Key words: ticks, integrated management, natural extract, Boophilus microplus, Costa Rica.

Las garrapatas duras (Ixodidae) se encuentran ampliamente distribuidas en las áreas tropicales, subtropicales y templadas del planeta, donde son un serio problema económico y de salud pública. En Costa Rica y el resto de América Latina, la especie más relevante para la ganadería bovina es Boophilus microplus Canestrini, 1887, debido a su amplia distribución, a los daños que ocasiona y a la transmisión de enfermedades (Anónimo 1980, Nari 1995, Álvarez et al. 1999).

Diferentes métodos han sido propuestos para el control de garrapatas, pero es el químico el que logró la mayor aceptación por las facilidades que presenta, su efecto rápido y por el poder económico de las transnacionales (Nari 1990, de Castro 1997). La intensificación de tales prácticas alcanza su mayor apogeo en la segunda mitad del siglo XX; sin embargo, una serie de aspectos han sido cuestionados en relación con el uso indiscriminado de los químicos sintéticos, tales como la contaminación, el efecto sobre las poblaciones no blanco, los peligros en la salud pública y el desarrollo de resistencia a esas sustancias por parte de las poblaciones de garrapatas (Panella et al. 1997, Kemp et al. 1998).

Ante esta situación, se han investigado en distintas partes del mundo diferentes opciones para el control de las garrapatas, por medio de las cuales se logró demostrar la acción biocida o biostática, con una serie de agentes controladores, entre otros, los entomopatógenos, los cuales presentan una enorme posibilidad para el manejo integrado de esta importante plaga (Lezama-Gutiérrez et al. 1995, Kocan et al. 1998, Samish et al. 1998, Zhioua et al. 1999). Asimismo otros investigadores han evaluado sustancias de origen vegetal y han encontrado actividad importante en el control de algunas de 
las especies de importancia pecuaria (Olusegun y Ndungu 1991, Panella et al. 1997, Monteiro et al. 1998, Huerta-Paniagua et al. 1999a, Huerta-Paniagua et al. 1999b).

Dentro de las metodologías que se han utilizado para verificar la actividad biológica de extractos de plantas, está la de los ensayos biodirigidos, la cual permite direccionar su utilización, conforme se van obteniendo los resultados preliminares.

Inicialmente las evaluaciones biológicas se realizan con extractos crudos hidroalcohólicos, a una máxima concentración. En este tipo de extracto (R-OH) se supone están contenidas aquellas moléculas o sustancias que por su potencial genético y fenotípico la planta puede producir, acumular o exhibir; y además, puedan manifestar alguna actividad biológica. Como siguiente paso, e independientemente de los resultados, los extractos crudos se particionan con un solvente de polaridad intermedia (diclorometano), con lo que se obtiene una parte polar y otra parte no polar. Esto se realiza para conjuntar los posibles grupos de metabolitos y verificar nuevamente la actividad biológica que alguna de las dos particiones pueda manifestar.

La acción de las sustancias garrapaticidas puede ser evaluada por su efecto sobre las poblaciones adultas o incluir otros estadíos. Un ejemplo de lo anterior son los inhibidores de desarrollo y los reguladores de crecimiento (Nari 1990, Blagburn y Lindasy 1995). Otros indicadores reproductivos de gran valor en las diferentes mediciones de las poblaciones de garrapatas son la oviposición y la eclosión.

El objetivo de esta investigación fue evaluar el efecto biocida o biostático sobre diferentes estados de desarrollo de las garrapatas B. microplus, mediante ensayos biodirigidos de extractos hidroalcohólicos (R-OH) y particiones (polares y no polares) de diferentes plantas.

\section{MATERIALES Y MÉTODOS}

Material biológico: se utilizaron garrapatas B. microplus para infestar terneros Bos taurus de 4-6 meses de edad, en condiciones de aislamiento en establo. Las garrapatas repletas se desprendían por si solas, eran recolectadas, lavadas y una vez revisadas se seleccionaban para evitar individuos malformados o dañados. Estas se pesaban y se homogenizaban en cuanto a peso para que no existieran diferencias mayores a $20 \mathrm{mg}$ entre cada una de las repeticiones para hacer las comparaciones. Todas las teleóginas se obtuvieron de infestaciones artificiales sobre animales estabulados.

Extractos vegetales: se seleccionaron diez plantas promisorias con base en revisiones bibliográficas e investigaciones realizadas con anterioridad y además se incluyó la evaluación de un producto comercial a base de canela (Weniger y Robineau 1986, Echeverri 1988, Munich 1988, Sagreda 1991, Bhushan 1992, Mejía 1997, Frenk et al. 2001). Se recolectó el material vegetal, el cual fue identificado desde el punto de vista taxonómico; después de secado y molido cada parte se colocó a extracción utilizando como solvente ethanol al $95 \%$ y agua, en una relación de 80:20, respectivamente. Quince días después se filtró el líquido y se concentró en un rotavapor hasta obtener el primer extracto $\mathrm{R}-\mathrm{OH}$. Como primer paso se evaluaron los extractos hidroalcohólicos (R-OH) obtenidos de las plantas mostradas en el Cuadro 1.

Una vez evaluada la actividad de los extractos crudos, se seleccionaron de acuerdo con los resultados, continuando con los más promisorios (aquellos que mostraron un efecto superior al $50 \%$ de la actividad). Posteriormente, los extractos R-OH se particionaron utilizando diclorometano, solvente de polaridad intermedia; con el que se obtuvieron dos fracciones, una polar y otra no polar, a las que se les eliminó el solvente en un rotavapor con el fin de realizar evaluaciones biológicas.

Ensayos in vitro: se realizó un tamizaje de las sustancias para determinar cuáles tenían un potencial más promisorio como acaricidas. En el ensayo se determinaron los siguientes parámetros: mortalidad de teleóginas, peso de oviposición, porcentaje de eclosión $(\% \mathrm{E})$ y 
CUADRO 1

Plantas evaluadas para el control de las garrapatas

TABLE 1

Plants evaluated for tick control

$\begin{array}{lllll}\text { Código } & \text { Nombre científico } & \text { Nombre vulgar } & \text { Familia } & \text { Parte de la planta utilizada } \\ \text { LOA 003 } & \text { Piper nigrum } & \text { Pimienta negra } & \text { Piperaceae } & \text { Espigas } \\ \text { LOA 009 } & \text { Zizygium aromaticum } & \text { Clavo de olor } & \text { Myrtaceae } & \text { Botones florales } \\ \text { LOA 015 } & \text { Petiveria aliaceae } & \text { Zorrillo, anamú } & \text { Phytolaccaceae } & \text { Raíz } \\ \text { LOA 018 } & \text { Echinacea angustifolia } & \text { Echinacea } & \text { Asteraceae } & \text { Raíz } \\ \text { LOA 022 } & \text { Piper aduncum } & \text { Alcotam } & \text { Piperaceae } & \text { Hojas } \\ \text { LOA 026 } & \text { Capsicum } \text { sp. } & \text { Chile picante } & \text { Solanaceae } & \text { Frutos } \\ \text { LOA 027 } & \text { Gliricidia sepium } & \text { Madero negro } & \text { Leguminoceae } & \text { Raíz } \\ \text { LOA 031 } & \text { Poligonum punctatum } & \text { Chile perro } & \text { Polygonaceae } & \text { Estolón } \\ \text { LOA 046 } & \text { Morus alba } & \text { Morera } & \text { Moraceae } & \text { Raíz } \\ \text { LOA 058 } & \begin{array}{l}\text { Allium sativum }- \text { Z. aromaticum } \\ \text { (50:50) }\end{array} & \text { Ajo x clavo de olor } & \text { Liliaceae y myrtaceae } & \text { Fruto y botones florales } \\ \text { 101 } & \text { Cinnamomum zeylanicum } & \text { Canela } & \text { Lauraceae } & \text { Producto comercial }\end{array}$

para algunas de las sustancias evaluadas, fue posible determinar el porcentaje de inhibición de la oviposición (\%IO) y el porcentaje de control $(\% \mathrm{C})$. Después de establecer cuáles eran las sustancias que manifestaban los mejores resultados, se prepararon diluciones 1:1 con las mejores de ellas, para estimar la actividad potencial de dicha dilución.

Se colocaron en cada ensayo grupos de 30-40 garrapatas, divididas en subgrupos de diez en placas de petri, las cuales eran seleccionadas y pesadas según los criterios expuestos anteriormente. A continuación se aplicó la prueba de inmersión de adultas, según Drummond (1967). Una vez expuestas al producto se colocaban en una estufa a una temperatura $(\mathrm{T})$ de $28{ }^{\circ} \mathrm{C}\left( \pm 1^{\circ} \mathrm{C}\right)$ y una humedad relativa (HR) de $95 \%$. Con las hembras repletas de los grupos control se efectuaba el mismo procedimiento pero en agua corriente y posteriormente se colocaban bajo las mismas condiciones de $\mathrm{T}$ y HR citadas. La mortalidad diaria se anotó en una plantilla diseñada para tal propósito hasta el día diez pos tratamiento.
El día 11 posterior al inicio de oviposición, se sacaba la teleógina y se pesaban los huevos, los cuales se colocaban en un vial tapado con algodón, el cual se colocaba en las mismas condiciones de $\mathrm{T}$ y HR arriba descritas. Una vez eclosionadas las larvas se eliminaban por medio de calor $\left(45^{\circ} \mathrm{C}\right)$ y se establecía el porcentaje de eclosión, asumiendo el dato aceptado de que en un gramo hay 20000 huevos. Todos estos datos se introducían en una base de datos para su posterior análisis.

Análisis de los datos: los datos fueron almacenados en un archivo de EpiInfo Versión 6.04b (Center for disease Control (CDC). World Health Organization). Las variables de respuesta fueron las siguientes: porcentaje de mortalidad, oviposición, \%E, porcentaje de control $(\% \mathrm{C})$ y $\%$ de Inhibición de la oviposición $(\% \mathrm{IO})$. Luego estas variables fueron transformadas a eficacia en mortalidad (EM), eficacia en la reducción de la oviposición (ERO) y eficacia en la reducción de la eclosión (ERE) según las directrices regulatorias 
europeas para preparaciones ectoparasíticas (Thrusfield et al. 1997).

Para la primera modificación y luego de los diez días de tratamiento, se calculó previamente la supervivencia de garrapatas, tanto de los controles como de los grupos experimentales, de la siguiente manera:

Supervivencia $=($ Garrapatas vivas/total garrapatas $) \times 100$

Luego se calculó la eficacia de la mortalidad del grupo control y de cada tratamiento con la siguiente fórmula:

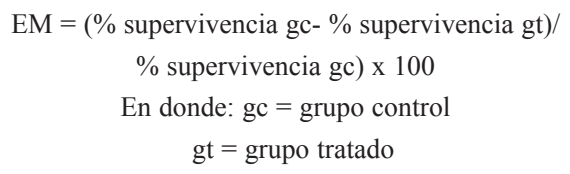

La eficacia de los diferentes extractos fue evaluada a través de tres parámetros: la mortalidad de teleóginas de B. microplus; la reducción de la oviposición (reducción de la relación huevos garrapatas) y la disminución de la eclosión.

La eficacia fue calculada mediante la siguiente fórmula:

$$
\% \text { eficacia }=\mathrm{C}-\mathrm{T} / \mathrm{Cx} 100
$$

En donde:

$\mathrm{C}=$ promedio o porcentaje del grupo control $\mathrm{T}=$ promedio o porcentaje del grupo tratado

El porcentaje de eclosión se estableció por medio de la siguiente fórmula:

$$
\% \mathrm{E}=\mathrm{C} / \mathrm{H}+\mathrm{Cx} 100
$$

En donde: $\mathrm{C}=$ cascarones, $\mathrm{H}=$ huevos

Para calcular la eficacia en la reducción de la oviposición, se obtuvo primero el número de huevos por gramo de garrapata $(\mathrm{HGG}=$ peso de huevos/peso de garrapatas)*20 000 de cada ensayo. Luego se calculó la ERO con la fórmula:

\section{$\mathrm{ERO}=((\mathrm{HGG}$ gc $-\mathrm{HGG}$ gt $) / \mathrm{HGG}$ gc $) / 100$}

Para calcular la ERE primero se obtuvo el porcentaje de huevos eclosionados por gramo de garrapata (\% HEPGG) de la siguiente forma:

$$
\left(\mathrm{HGG}^{*} \% \text { eclosión }\right) / 100
$$

Luego se calculó la ERE $=((\%$ HEPGG gc $\%$ HEPGG gt) $/ \%$ HEPGG gc)*100.

Una vez calculados la EM, la ERO y la ERE, se realizaron comparaciones de cada una de las proporciones calculadas.

Los cálculos de $\%$ IO y \% C se hicieron según se indica en el Curso Taller sobre Diagnóstico de Resistencia a Ixodicidas en garrapatas Boophilus microplus (Anónimo 2001).

\section{RESULTADOS}

Efecto de los extractos sobre la mortalidad: los resultados de eficacia de los diferentes extractos R-OH y sus diluciones, sobre la mortalidad de garrapatas repletas B. microplus se observan en el Cuadro 2.

En este grupo sobresale el efecto del extracto 101 (canela) el cual expresó un $100 \%$ de mortalidad a su concentración máxima; sin embargo, la actividad disminuyó casi totalmente $(2.18 \%)$ cuando se evaluó en una dilución 1:1 en agua. Pudo haber un enmascaramiento o antagonismo de aquel o aquellos mebatolitos que inicialmente manifestaron actividad los cuales al hacer la dilución, por algún efecto de concentración, fueron bloqueados o diluidos. Otro extracto, que manifestó un buen porcentaje de mortalidad (68\%), fue el 046 (morera); el cual además presentó una tendencia esperada, ya que el porcentaje de inhibición de la mortalidad fue de $36 \%$ cuando se evaluó en una dilución de 1:1 partes de agua, respectivamente.

Los resultados de la eficacia de las particiones (polar y no polar) sobre la mortalidad se presentan en la Fig. 1 y 2. En la Fig. 1, donde se agrupan los mejores resultados de los extractos y sus diluciones polares, ninguno de 
CUADRO 2

Eficacia de los extractos vegetales $(\mathrm{ROH})$ in vitro sobre la mortalidad de teleóginas de $\mathrm{B}$. microplus

TABLE 2

Effectiveness of the plant extracts $(\mathrm{ROH})$, in vitro, on the mortality of adult $\mathrm{B}$. microplus females

\begin{tabular}{|c|c|c|c|c|c|}
\hline Extracto & Dilución & Ensayos & $\begin{array}{c}\text { Garrapatas } \\
\text { Totales }\end{array}$ & $\begin{array}{c}\text { Porcentaje } \\
\text { eficacia }\end{array}$ & Valor $\mathrm{p}^{1}$ \\
\hline 003 & Pura & 9 & 96 & 22.74 & $\mathrm{~d}$ \\
\hline 003 & $1: 1$ & 5 & 50 & 40.28 & $\mathrm{c}$ \\
\hline 015 & Pura & 8 & 80 & 23.72 & $\mathrm{~d}$ \\
\hline 015 & $1: 1$ & 5 & 50 & 3.64 & $\mathrm{e}$ \\
\hline 018 & Pura & 9 & 90 & 31.62 & $\mathrm{~cd}$ \\
\hline 018 & $1: 1$ & 5 & 50 & 4.95 & $\mathrm{e}$ \\
\hline 022 & Pura & 9 & 90 & 15.32 & de \\
\hline 022 & $1: 1$ & 5 & 50 & 1.31 & $\mathrm{f}$ \\
\hline 026 & Pura & 8 & 80 & 11.22 & $\mathrm{e}$ \\
\hline 026 & $1: 1$ & 5 & 50 & 3.64 & ef \\
\hline 027 & Pura & 8 & 80 & 9.75 & ef \\
\hline 027 & $1: 1$ & 5 & 50 & 7.30 & ef \\
\hline 046 & Pura & 4 & 40 & 67.88 & $\mathrm{~b}$ \\
\hline 046 & $1: 1$ & 4 & 40 & 35.75 & $\mathrm{~cd}$ \\
\hline 101 & Pura & 5 & 50 & 100 & a \\
\hline 101 & $1: 1$ & 9 & 90 & 2.18 & $\mathrm{f}$ \\
\hline 003 & Pura & 9 & 96 & 22.74 & $\mathrm{~d}$ \\
\hline 003 & $1: 1$ & 5 & 50 & 40.28 & $\mathrm{c}$ \\
\hline 015 & Pura & 8 & 80 & 23.72 & $\mathrm{~d}$ \\
\hline 015 & $1: 1$ & 5 & 50 & 3.64 & e \\
\hline 018 & Pura & 9 & 90 & 31.62 & $\mathrm{~cd}$ \\
\hline 018 & $1: 1$ & 5 & 50 & 4.95 & e \\
\hline 022 & Pura & 9 & 90 & 15.32 & de \\
\hline 022 & $1: 1$ & 5 & 50 & 1.31 & $\mathrm{f}$ \\
\hline 026 & Pura & 8 & 80 & 11.22 & e \\
\hline 026 & $1: 1$ & 5 & 50 & 3.64 & ef \\
\hline 027 & Pura & 8 & 80 & 9.75 & ef \\
\hline 027 & $1: 1$ & 5 & 50 & 7.30 & ef \\
\hline 046 & Pura & 4 & 40 & 67.88 & b \\
\hline 046 & $1: 1$ & 4 & 40 & 35.75 & $\mathrm{~cd}$ \\
\hline 101 & Pura & 5 & 50 & 100 & $\mathrm{a}$ \\
\hline 101 & $1: 1$ & 9 & 90 & 2.18 & $\mathrm{f}$ \\
\hline
\end{tabular}

1 Las letras significan diferencias estadísticas entre los valores de eficacia. 


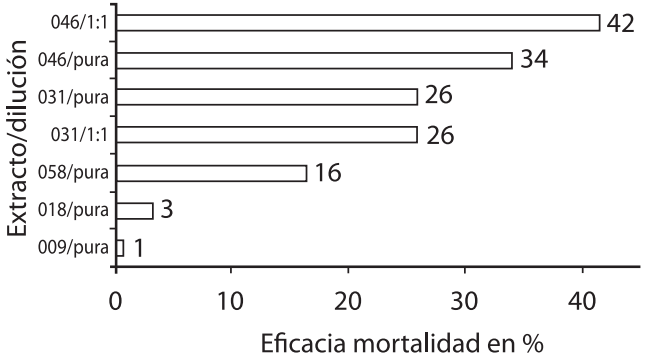

Fig. 1. Eficacia de extractos vegetales polares in vitro en mortalidad de teleóginas de B. microplus.

Fig. 1. Effectiveness of polar plant extracts, in vitro, on mortality of adult $B$. microplus females.

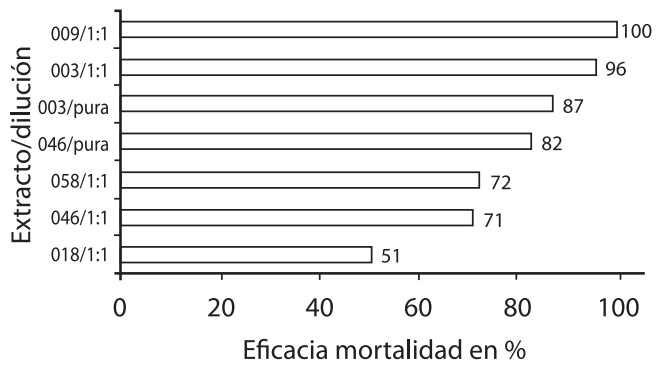

Fig. 2. Eficacia de extractos vegetales no polares in vitro en mortalidad de teleóginas de B. microplus.

Fig. 2. Effectiveness of no polar plant extracts, in vitro, on mortality of adult $B$. microplus females.

ellos alcanzó una actividad promisoria (igual o mayor al $50 \%$ ) sobre la mortalidad de teleóginas.

En la Fig. 2 se agrupan los resultados de los extractos no polares y se encontró que el 003/1:1 (pimienta negra) y el 009/1:1 (clavo de olor) presentaron la eficacia más alta, aunque no mostraron diferencias estadísticas significativas entre sí; no obstante, si hubo diferencias estadísticas con los demás extractos analizados. La eficacia de los extractos 003/puro y 046/ puro fue igual entre ambos, pero diferente a los demás. La eficacia menor se detectó en los extractos 058/1:1 (mezcla de ajos y clavo de olor) y 046/1:1. En los demás extractos y concentraciones estudiados la eficacia fue menor del $50 \%$.
Efecto de los extractos sobre la reducción de la oviposición: en el Cuadro 3 se observa que los extractos $\mathrm{R}-\mathrm{OH}$ puros y sus diluciones 1:1, tanto del 046 (morera) y del 003 (pimienta negra) manifestaron una actividad promisoria de 74 y $57 \%$; y $66 \%$ y $44 \%$ respectivamente, sobre la oviposición de teleóginas de $B$. microplus, con lo cual se constituyen como los más promisorios y con una tendencia esperada según el efecto de dilución. No se encontraron diferencias significativas entre ellos. La actividad ovicida de los extractos polares se presenta en la Fig. 3 en la cual, solo el extracto polar 046 (morera) en la dilución 1:1 mostró un resultado superior al $50 \%$ de efectividad.

En la Fig. 4, por el contrario, se observa que una serie de extractos y sus diluciones mostraron un efecto ovicida superior al $50 \%$. Los extractos 003/1:1, 058/1:1, 003/puro y 046/

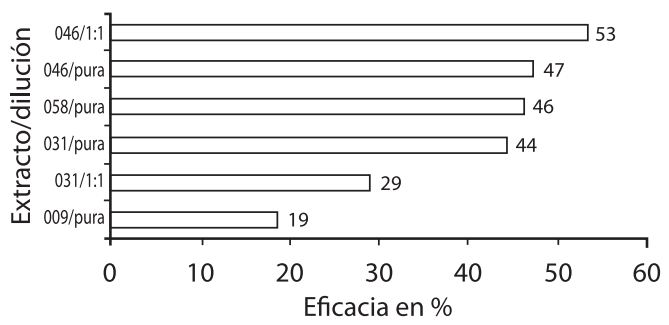

Fig. 3. Eficacia de extractos vegetales polares in vitro en reducción de oviposición de $B$. microplus.

Fig. 3. Effectiveness of polar plant extracts, in vitro, on the reduction of $B$. microplus oviposition.

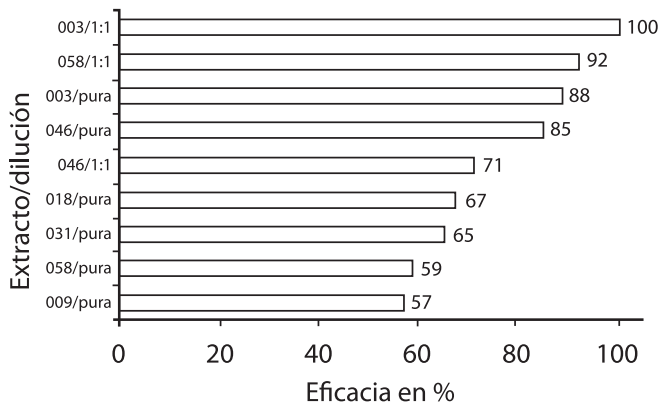

Fig. 4. Eficacia de los extractos vegetales no polares en la reducción de la oviposición de $B$. microplus.

Fig. 4. Effectiveness of nonpolar vegetal extracts in the reduction of $B$. microplus oviposition. 
CUADRO 3

Eficacia de los extractos vegetales $\mathrm{R}-\mathrm{OH}$ in vitro en reducción de la oviposición de $\mathrm{B}$. microplus

TABLE 3

Effectiveness of $\mathrm{R}-\mathrm{OH}$ plant extracts, in vitro, on reduction of $\mathrm{B}$. microplus oviposition

\begin{tabular}{|c|c|c|c|c|c|}
\hline Extracto & Dilución & Ensayos & Garrapatas & Porcentaje eficacia & Valor $\mathrm{p}^{1}$ \\
\hline 003 & Pura & 9 & 96 & 65.60 & a \\
\hline 003 & $1: 1$ & 4 & 40 & 43.83 & $\mathrm{~b}$ \\
\hline 015 & Pura & 8 & 80 & 28.50 & $\mathrm{bc}$ \\
\hline 015 & $1: 1$ & 5 & 50 & 18.70 & $\mathrm{c}$ \\
\hline 018 & Pura & 9 & 90 & 33.31 & $\mathrm{~b}$ \\
\hline 018 & $1: 1$ & 5 & 50 & 12.12 & $\mathrm{c}$ \\
\hline 022 & Pura & 9 & 90 & 34.10 & $\mathrm{~b}$ \\
\hline 022 & $1: 1$ & 5 & 50 & 6.80 & $\mathrm{c}$ \\
\hline 026 & Pura & 8 & 80 & 15.38 & $\mathrm{c}$ \\
\hline 026 & $1: 1$ & 5 & 50 & 11.81 & $\mathrm{c}$ \\
\hline 027 & Pura & 8 & 80 & 16.60 & $\mathrm{c}$ \\
\hline 027 & $1: 1$ & 5 & 50 & 17.08 & $\mathrm{c}$ \\
\hline 046 & Pura & 4 & 40 & 74.37 & $\mathrm{a}$ \\
\hline 046 & $1: 1$ & 4 & 40 & 56.72 & $\mathrm{ab}$ \\
\hline 101 & $1: 1$ & 9 & 90 & 14.40 & $\mathrm{c}$ \\
\hline
\end{tabular}

1 Las letras significan diferencias estadísticas entre los valores de eficacia.

puro no tuvieron entre si diferencias estadísticas significativas, pero sí con el resto, el cual no mostró diferencias entre sí.

Efecto de los extractos sobre la reducción de la eclosión: según el Cuadro 4, no fueron localizadas moléculas o sustancias en la parte polar de los extractos probados que manifiesten una actividad promisoria para el control de la eclosión.

Una vez más, los resultados muestran una muy importante actividad sobre uno de los indicadores reproductivos de la garrapata en la parte no polar de los extractos probados. Según el Cuadro 5 la actividad promisoria sobre la eclosión se concentra en los extractos no polares $003,058,046,031,018$ y 009.

Porcentaje de control: para determinar el porcentaje de control de B. microplus, se utilizaron los extractos 003, 018, 031, $027 \mathrm{y}$ 046, los cuales fueron dispuestos en cuatro clases según el nivel de control (Fig. 5). En la clase de control de 76-100 \% se ubicó al extracto 003 con las particiones no polares desde pura hasta la dilución de 1:40; el extracto 018 no polar en las diluciones pura hasta 1:10; la 031 no polar en las concentraciones pura y 1:1 y la 046 no polar en las concentraciones pura, 1:20 y $1: 40$ y polar $1: 20$ y 1:40. En la clase $51-75 \%$ se ubicó a la partición no polar del extracto 046 en la concentración 1:1; a la partición $\mathrm{ROH}$ de la 046 en las concentraciones pura y $1: 1$; a la partición 031 polar en la concentración pura. El resto de particiones entre las que se encuentran polares, no polares y $\mathrm{ROH}$ de diferentes concentraciones si acaso alcanzaron el $50 \%$ de porcentaje de control. Entre las sustancias que están en esa situación hay extractos de las plantas 018, 031, 046 y 051. 
CUADRO 4

Eficacia de los extractos vegetales polares in vitro en la reducción de la eclosión de B. microplus

TABLE 4

Effectiveness of polar plant extracts, in vitro, on the reduction of $\mathrm{B}$. microplus hatchability

\begin{tabular}{|c|c|c|c|c|c|}
\hline Extracto & Dilución & Ensayos & Garrapatas & Porcentaje eficacia & Valor $\mathrm{p}^{1}$ \\
\hline 009 & Pura & 10 & 100 & 0 & $\mathrm{c}$ \\
\hline 009 & $1: 1$ & 10 & 100 & 0 & $\mathrm{c}$ \\
\hline 031 & Pura & 18 & 180 & 22.45 & a \\
\hline 031 & $1: 1$ & 18 & 180 & 8.14 & $\mathrm{~b}$ \\
\hline 046 & Pura & 4 & 40 & 0 & $\mathrm{~b}$ \\
\hline 046 & $1: 1$ & 4 & 40 & 0 & $\mathrm{~b}$ \\
\hline 058 & Pura & 7 & 70 & 18.73 & $\mathrm{a}$ \\
\hline 058 & $1: 1$ & 8 & 80 & 0 & $\mathrm{c}$ \\
\hline
\end{tabular}

1 Las letras significan diferencias estadísticas entre los valores de eficacia.

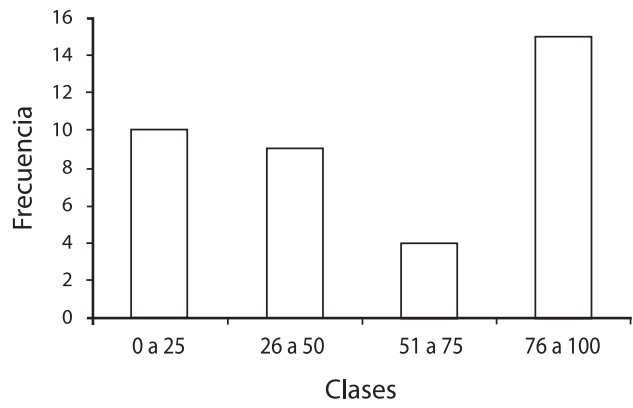

Fig. 5. Porcentaje de control de teleóginas, según extracto.

Fig. 5. Percentage of control over adult females, according to extract.

\section{Porcentaje de inhibición de la oviposi-} ción: para la prueba de inhibición de la oviposición se utilizaron 003, 018, 027, 031 y 046. Del extracto 003, se utilizó la partición no polar desde pura hasta la dilución de 1:80, de la partición polar de 1:40 a 1:80. Del extracto 018 solo se utilizó la partición no polar de pura hasta la dilución de 1:20. De los extractos 031,027 y 046 se utilizaron las dos particiones desde puras hasta 1:40. Además del extracto 046 se utilizó la ROH pura hasta 1:10.

Los primeros ocho extractos presentados en la Fig. 6, tuvieron resultados entre $99.7 \%$

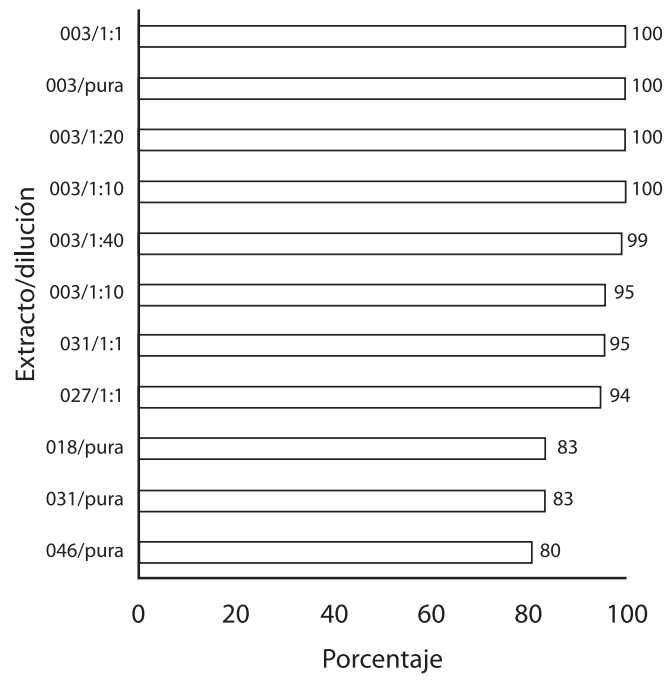

Fig. 6. Porcentaje de inhibición de la oviposición, según extracto y dilución.

Fig. 6. Percentage of oviposition inhibition, by extract and dilution.

y $94.4 \%$ de inhibición de oviposición; estos no presentaron diferencias estadísticas significativas entre ellos. Los extractos 018/pura, 031/pura y 046/pura presentaron resultados menores $(\mathrm{p}<0.05)$. 
CUADRO 5

Eficacia de los extractos vegetales no polares in vitro en la reducción de la eclosión de B. microplus

TABLE 5

Effectiveness of nonpolar plant extracts, in vitro, on the reduction of B. microplus hatchability

$\begin{array}{cccccc}\text { Extracto } & \text { Dilución } & \text { Ensayos } & \text { Garrapatas } & \text { Porcentaje eficacia } & \text { Valor } \mathrm{p}^{1} \\ 003 & \text { Pura } & 6 & 60 & 99.85 & \mathrm{a} \\ 009 & \text { Pura } & 9 & 90 & 75.93 & \mathrm{~b} \\ 018 & \text { Pura } & 28 & 280 & 81.79 & \mathrm{~b} \\ 031 & \text { Pura } & 16 & 160 & 84.44 & \mathrm{~b} \\ 031 & 1: 1 & 13 & 130 & 72.34 & \mathrm{~b} \\ 046 & \text { Pura } & 4 & 40 & 85.43 & \mathrm{~b} \\ 046 & 1: 1 & 4 & 40 & 71.88 & \mathrm{~b} \\ 058 & \text { Pura } & 8 & 80 & 79.18 & \mathrm{~b} \\ 058 & 1: 1 & 6 & 60 & 98.08 & \mathrm{a}\end{array}$

1 Las letras significan diferencias estadísticas entre los valores de eficacia.

De los resultados de inhibición de la oviposición obtenidos con los otros extractos, se pudieron diferenciar dos grupos, el primero con actividad intermedia de $77.15 \%$ a $50.95 \%$ y el segundo con actividad inferior de $44.4 \%$ a $0 \%$.

En el grupo de actividad intermedia se ubican los extractos 018 con la partición polar y dilución 1:1;027 partición no polar pura; 031 con la partición polar y dilución 1:1,046 con la partición no polar 1:1 y la fracción ROH pura. Este grupo presentó una diferencia estadística significativa con los extractos presentados en la Fig. $6(\mathrm{p}<0.05)$ y con los del grupo de actividad inferior $(\mathrm{p}<0.05)$.

\section{DISCUSIÓN}

Como se observa en el Cuadro 2 que los extractos hidroalcohólicos 101 y 046 a la máxima concentración mostraron una actividad mayor al $50 \%$ sobre la mortalidad de teleóginas. Esto permite inferir que en los extractos $\mathrm{R}-\mathrm{OH}$ de estas plantas están presentes moléculas o sustancias que ejercen algún efecto promisorio para el control de este parásito.
No es factible, según los objetivos del trabajo, determinar los motivos por los cuales la sustancia 003 en la dilución más débil, mostró un mejor resultado en cuanto a mortalidad; sin embargo, lo anterior pudo deberse a algún antagonismo o enmascaramiento producto de la presencia de diferentes moléculas o sustancias en el extracto crudo. En la partición polar 046 se ubicó una situación similar a la descrita anteriormente en la cual una concentración más débil manifiesta una acción más fuerte; sin embargo, estadísticamente no significó diferencia alguna.

En la literatura se reportan investigaciones realizadas en Colombia con Poligonum spp. y Capsicum spp. En el caso de Capsicum spp. para el parámetro mortalidad los resultados fueron superiores a los logrados en esta investigación; sin embargo, no alcanzaron el $50 \%$ de efectividad. En relación con Poligonum spp. los indicadores de mortalidad reportados en este trabajo fueron iguales a los obtenidos por los investigadores colombianos por medio del método Soxhlet (25\%). (Anónimo 2004).

Como paso siguiente de la investigación se evaluaron particiones de los extractos R-OH. 
La evaluación de particiones permite en ocasiones observar algún enmascaramiento o antagonismo de los metabolitos o bloqueo de otro tipo (resinas, taninos etc) que impide la manifestación de actividad en los extractos R-OH.

Los extractos polares concentran metabolitos secundarios de naturaleza polar. Según la Fig. 1, en esta partición no se encontraron moléculas o sustancias que mostraran actividad mayor al $50 \%$ de eficacia sobre la mortalidad; sin embargo, sí es clara la presencia, aunque evidentemente débil, de algunos metabolitos con cierto nivel de acción sobre las teleóginas.

Los resultados de las evaluaciones con las particiones no polares muestran, según la Fig. 2, que los extractos 009, 003, 046, 058, 018 evaluados a la concentración máxima mostraron actividad mayor al $50 \%$. Inclusive cuando se evaluaron diluciones de estos extractos, se obtuvieron resultados promisorios mayores al $50 \%$ de mortalidad.

Chungsamarnyart et al. (1991), describieron la actividad larvicida de un extracto alcohólico de Streblus asper Lour. una planta de la familia Moraceae, al igual que la morera (046), e indican que este tuvo un efecto relativamente alto sobre la mortalidad (71-85\%).

Chungsamarnyart et al. (1994), indicaron sobre la elevada pero lenta actividad acaricida de Piper nigrum (003) en combinación con otros extractos de plantas, mostrando un importante sinergismo. En el presente estudio los extractos fueron aplicados sin ningún sinergista o alguna otra sustancia que pudiera potencializar los metabolitos presentes en el extracto, a través de una mejor absorción como producto de una mayor permeabilización o de otro mecanismo de interiorización.

En comparación con las particiones polares, se observa que las principales moléculas con una actividad promisoria sobre la mortalidad, están presentes en la parte no polar de los extractos vegetales probados. Se nota que los extractos hidroalcohólicos 009, 003, 058, y 018 que no habían manifestado una actividad promisoria en su evaluación como R-OH, al realizar las particiones, presentaron un posible desenmascaramiento de los metabolitos responsables de la actividad, y de esa forma sí manifestaron una actividad importante en cuanto a la mortalidad de teleóginas $B$. microplus, particularmente las fracciones no polares.

En resumen, la mortalidad de teleóginas de B. microplus con algunos extractos probados mostró efectos importantes a tomar en cuenta, en especial las particiones no polares. Un nuevo paso en la evaluación de moléculas promisorias para el control de las garrapatas consiste en determinar el potencial de su capacidad ovicida. Nuevamente la evaluación de la capacidad ovicida de extractos vegetales se inicia con los extractos hidroalcohólicos R-OH, en los cuales están presentes todas las posibles moléculas acumuladas por la planta.

El efecto ovicida observado en la parte polar evaluada de estos extractos, muestra solamente actividad mayor al $50 \%$ para el extracto 046; sin embargo, otras particiones polares como la 031 y la 058 mostraron un potencial ovicida importante, con lo cual se podría esperar la presencia de algunas moléculas con acción sobre este parámetro reproductivo en esta parte de los extractos. El efecto o la actividad de mayor peso sobre este indicador reproductivo de las garrapatas se localiza en la parte no polar de los extractos de las plantas.

Los indicadores de $\% \mathrm{C} \mathrm{y} \% \mathrm{IO}$ fueron excelentes en algunas de las sustancias, particiones y concentraciones probadas. Los resultados con el Poligonum spp. obtenidos en el presente estudio, son muy superiores a los conseguidos por los investigadores de Corpoica (2004).

Una serie de extractos utilizados en el presente estudio, mostraron actividad promisoria sobre al menos algún parámetro de referencia importante en la vida de las garrapatas $B$. microplus; sin embargo, no fue posible realizar una mayor discusión sobre los diferentes extractos obtenidos de varias plantas debido a que no se cuenta con información previa sobre el efecto de estas.

Los resultados muestran de forma clara el efecto in vitro de varias sustancias de origen vegetal sobre distintos estadios del desarrollo de las garrapatas B. microplus, lo que provocó mortalidad en los adultos y disminuyó, en 
algunos casos de manera sensible la oviposición o provocaron una merma considerable en el porcentaje de eclosión de los huevos depositados por las teleóginas.

Es necesario por tanto, profundizar aún más estos resultados preliminares para poder conocer cuáles podrían ser las moléculas implicadas, el efecto in vivo sobre las poblaciones de garrapatas, el efecto sobre estadíos inmaduros, la persistencia de los diferentes metabolitos, las dosis letales $\left(\mathrm{DL}_{50}\right)$, etc., sin olvidar aspectos ambientales como el efecto sobre poblaciones no blanco y, por supuesto, lo relacionado con los residuos en productos de consumo humano.

\section{AGRADECIMIENTOS}

A José Cascante por su apoyo en el laboratorio. A Jorge Briceño y Marco Herrero por sus comentarios. A Sara Valverde por su revisión de estilo. A Dagoberto Méndez por su colaboración.

\section{RESUMEN}

Usando el método de inmersión, probamos el efecto de extractos vegetales hidroalcohólicos de diez plantas tropicales en garrapatas adultas (Boophilus microplus Canestrini 1887). Usamos extractos crudos hidroalcohólicos (R-OH) para determinar los compuestos más promisorios, los que posteriormente se fraccionaron en sus partes polares y no polares. Las particiones polares de los extractos vegetales mostraron un efecto significativamente inferior que las no polares. Las mayores mortalidades se lograron con: Zizygium aromaticum, Morus alba, Piper nigrum y la mezcla de Allium sativum - Z. aromaticum (todos no polares); sobre la oviposición $M$. alba, $P$. nigrum y la mezcla de $A$. sativum-Z. aromaticum (todos no polares); sobre la eclosión P. nigrum, Z. aromaticum, Echinacea angustifolia, Poligonum punctatum, M. alba y la mezcla de A. sativum-Z. aromaticum (todos no polares); sobre el porcentaje de control P. nigrum, E. angustifolia, $P$. punctatum y $M$. alba (todos no polares, con excepción de $M$. alba en el cual ambas fracciones mostraron actividad importante); sobre el porcentaje de inhibición de la oviposición P. nigrum, E. angustifolia, P. punctatum, Gliricidia sepium y M. alba.

Palabras clave: garrapatas, manejo integrado, extractos naturales, Boophilus microplus, Costa Rica.

\section{REFERENCIAS}

Álvarez, V., R. Bonilla \& I. Chacón. 1999. Determinación de la resistencia de la garrapata Boophilus microplus (Acari: Ixodidae) a organofosforados y piretroides en Costa Rica. Rev. Cien. Vet. 22: 41-60.

Anónimo. 1980. Informe Final Proyecto Estudio de Factibilidad para el Control de la Garrapata. Ministerio de Agricultura y Ganadería, Dirección de Salud Animal, San José, Costa Rica.

Anónimo. 2001. Cálculo de parámetros biológicos de la prueba biológica y bioensayos con concentraciones múltiples (Probit), p. 33-34. In M. Santamaría \& N. Soberanes (eds.). Curso-Taller sobre Diagnóstico de Resistencia a Ixodicidas en Garrapatas Boophilus microplus. SAGARPA, Jiutepec, Morelos, México.

Anónimo. 2004. Corpoica. Informe Técnico. Evaluación y Validación de la Medicina Herbaria en el Control de Parásitos Externos de Bovinos para Pequeños Productores de las Zonas de Ladera Cálida y Media de los Departamentos del Cauca y del Valle del Cauca. Corpoica, Palmira, Valle del Cauca, Bogotá, Colombia.

Bhushan, M.N. 1992. Handbook of Natural Pesticides: Methods, Vol. II Isolation and Identification. CRC, Boca Ratón, Florida, EEUU.

Blagburn, B. \& D. Lindsay. 1995. Ectoparasiticides, p. 984-1003. In H. Richard Adams (ed.). Veterinary Pharmacology and Therapeutics. Iowa State University, Ames, Iowa, EEUU.

Chungsamarnyart, N., S. Jiwajinda \& W. Jansawan. 1991. Larvicidal Effect of Plant Crude-extracts on the Tropical Cattle Tick (Boophilus microplus). Kasetsart J. (Nat. Sci. Suppl.) 25: 80-89.

Chungsamarnyart, N., C. Ratanakreetakul \& W. Jansawan. 1994. Acaricidal Activity of the Combination of Plant Crude-extracts to Tropical Cattle Tick. Kasetsart J. (Nat. Sci.) 28: 649-660.

De Castro, J. 1997. Sustainable tick and tickborne disease control in livestock improvement in developing countries. Vet. Parasitology 71: 77-97.

Drummond, R.O., O.H. Graham, S.E. Ernst \& J.L. Treviño. 1967. Evaluation of insecticides for the control of Boophilus annulatus (Say) and Boophilus microplus (Canestrini) (Acari: Ixodidae) on cattle, p. 493498. In Proccedings, $2^{\text {nd }}$ International Congress of Acarology. Akademia Kiado, Budapest, Hungría. 
Echeverri, L.F. 1988. Productos naturales biológicamente activos. Universidad de Antioquia, Facultad de Ciencias Exactas y Naturales, Centro de Investigaciones, Medellin, Colombia.

Frenk, M.J., B.R. Ruelas, C.R. Tapia, R.R. Castañon \& G.A. Lifshitz. 2001. Farmacopea Herbolaria de los Estados Unidos Mexicanos. Secretaria de Salud, Comisión permanente de la Farmacopea de los Estados Unidos Mexicanos, México D.F., México.

Huerta-Paniagua, R.A., C. Rodríguez-Hernández \& J.A.S. Villagómez-Cortés. 1999a. Evaluación de la actividad del cundeamor (Momordica charantia L.) (Cucurbitaceae) sobre Boophilus microplus (Canestrini), p. 120. In G. Morales, H. Fragoso \& Z. García (eds.). Cuarto Seminario Internacional de Parasitología Animal, Pto. Vallarta, Jalisco, México.

Huerta-Paniagua, R.A. \& C. Rodríguez-Hernández. 1999b. Evaluación de la actividad de extractos acuosos del nim (Azadirachta indica (L.) Juss.) (Meliaceae) sobre Boophilus microplus (Canestrini), p. 121. In G. Morales, H. Fragoso \& Z. García (eds.). Cuarto Seminario Internacional de Parasitología Animal, Pto. Vallarta, Jalisco, México.

Kemp, D., F. Thullner, K. Gale, A. Nari \& G. Sabatini. 1998. Report to the Animal Health Services, AGAH, FAO, CSIRO, Sidney, Australia.

Kocan, K.M., E.F. Blouin, M.S. Pidherney, P.L. Claypool, M. Samish \& I. Glazer. 1998. Entomopathogenic nematodes as a potential biological control method for ticks, p. 355-364. In F. Jongejan, W. Goff \& E. Camus (eds.). Tropical Veterinary Medicine. Molecular epidemiology, hemoparasites and their vectors, and general topics. Society for Tropical Veterinary Medicine, Nueva York, Nueva York, EEUU.

Lezama-Gutiérrez, R., E. Galindo-Velazco \& F. CruzCobian. 1995. Effectiveness of the entomopathogenic fungus Metarhizium anisopliae in the biological control of ticks in bovine livestock, in field conditions, p. 145. In S. Rodríguez \& H. Fragoso (eds.). Tercer Seminario Internacional de Parasitología Animal, Acapulco, Guerrero, México.

Mejía, G.M. 1997. Saber popular y medicina veterinaria herbolaria. Mi nuevo Mundo, Corporación para la Educación Especial, Cali, Colombia.

Monteiro, S.G., M.E. Carneiro, V.R.E.P. Bittencourt \& E. Daemon. 1998. Efeito do isolado 986 do fungo
Beauveria bassiana (Bals) Vuill sobre fêmeas ingurgitadas de Anocentor nitens Neumann, 1897 (Acari: Ixodidae). Arq. Bras. Med. Vet. Zootec. 50: 673-376.

Munich, L.E. 1988. Plantas con Propiedades Plaguicidas. Centro de Documentación CATIE-MIP-DPV, Turrialba, Alajuela, Costa Rica.

Nari, A. 1990. Methods currently used for the control of one-host ticks: their validity and proposals for future control strategies. Parassitologia 32: 133-143.

Nari, A. 1995. Strategies for the control of one-host ticks and relationship with tick-borne diseases in South America. Vet. Parasitol. 57: 153-165.

Olusegun, D. \& J.N. Ndungu. 1991. Acaricidal activity of Kupetaba, a ground mixture of natural products, against Rhipicephalus appendiculatus. Vet. Parasitology 38: 327-338.

Panella, N.A., J. Karchesy, G.O. Maupin, J.C.S. Malan \& J. Piesman. 1997. Susceptibility of inmature Ixodes scapularis (Acari: Ixodidae) to plant-derived acaricides. J. Med. Entomol. 34: 340-345.

Sagreda, F.J. 1991. Enciclopedia de Medicina Natural: Medicinas Alternativas. Zamora, Bogotá, Colombia.

Samish, M., E. Alekseev \& Y. Glazer. 1998. The effect of soil composition on anti-tick activity of entomopathogenic nematodes, p. 402-403. In Tropical Veterinary Medicine. Molecular epidemiology, hemoparasites and their vectors, and general topics. Society for Tropical Veterinary Medicine, Nueva York, Nueva York, EEUU.

Thrusfield, M.V. 1997. The Design and Conduct of Clinical Trials. Defining Efficacy. Efficacy of ectoparasitic preparations, p. 225-246. In J.P.T.M. Noordhuizen, K. Frankena, C.M. Van der Hoofd \& E.A.M. Graat. Application of Quantitative Methods in Veterinary Epidemiology, Wageningen, Holanda.

Weniger, B. \& L. Robineau. 1986. Investigaciones Científicas y uso popular de plantas medicinales en el Caribe. Seminario TRAMIL, Santo Domingo, República Dominicana.

Zhioua, E., K. Heyer, H. Ginsberg \& R. Lebrun. 1999. Control microbiológico de garrapatas, p. 177. In G. Morales, H. Fragoso \& Z. García (eds.). Cuarto Seminario Internacional de Parasitología Animal, Pto. Vallarta, Jalisco, México. 